\title{
Electronic reconstruction and surface two-dimensional electron gas in a polarized heterostructure with a hole-doped single copper-oxygen plane
}

\author{
Xiaoping Yang and Haibin $\mathrm{Su}^{*}$ \\ Division of Materials Science, Nanyang Technological University, 50 Nanyang Avenue, 639798 Singapore and \\ Institute of Advanced Study, Nanyang Technological University, 60 Nanyang View, 639673 Singapore
}

(Received 13 February 2012; revised manuscript received 9 August 2012; published 13 May 2013)

\begin{abstract}
We show that confinement together with intrinsic polarization induce hole doping of the $\mathrm{CuO}_{2}$ plane in the proposed $\mathrm{SrTiO}_{3} /\left[\mathrm{SrO}-\mathrm{La}_{2} \mathrm{CuO}_{4}-\mathrm{SrO}\right] / \mathrm{LaAlO}_{3}$ heterostructure without resorting to chemical substitution, using the generalized gradient approximation and its combination with Coulomb correlation $U$. Hole concentration can be rationally manipulated via tuning the $\mathrm{LaAlO}_{3}$ thickness and in-plane strain. The stable antiferromagnetic insulating bulk state up to $8 \%$ doping in this disorder-free structure is remarkable; moreover, this antiferromagnetic state coexists with two-dimensional electron gas on the top surface of $\mathrm{LaAlO}_{3}$, which originates from out-of-plane charge transfer mainly between the La- $d_{3 z^{2}-1}$ state at the surface and Cu- $e_{g}$ states in the $\mathrm{CuO}_{2}$ plane. In addition, a possible $\mathrm{SrTiO}_{3}$ capping layer is introduced in which, instead of $\mathrm{La}-d_{3 z^{2}-1}$, $\mathrm{Ti}-t_{2 g}$ orbitals exchange holes with electrons of $\mathrm{Cu}-e_{g}$ orbitals.
\end{abstract}

DOI: 10.1103/PhysRevB.87.205116

PACS number(s): 71.20.-b, 71.30.+h, 75.47.Lx

In the past decades, searching microscopic mechanism for superconductivity in cuprates remains central to hightemperature superconductivity (SC) research. ${ }^{1}$ It is identified that the electronic origin from the partially occupied $d_{x^{2}-y^{2}}$ orbital of $\mathrm{CuO}_{2}$ planes with antiferromagnetic (AFM) correlations plays a critical role in the peculiar phenomena discovered in the undoped parent compounds. ${ }^{2}$ However, the $\mathrm{SC}$ mechanism is still elusive; in particular the evolution from AFM to non-Fermi liquid is arguably attributed to the disorder dopants, ${ }^{3}$ whose microscopic origin is under debate due to the complexity and diversity of the chemical doping process.

The recent development of the oxide heterostructure (HS) interface technology offers a new platform to study the detailed physical properties of correlated oxide materials, because the interface can be used to modulate the electronic structure for manipulating physical properties and generating novel phases which are not present in the bulk constituents, especially through electronic and orbital reconstruction. ${ }^{4-6}$ Experimentally, Jang et al. found that electronic correlation and electronic structure of oxide HSs can be tuned by inserting a single atomic layer of different rare-earth oxides at the interface. ${ }^{6}$ In particular, Bozovic et al. have fabricated heterojunctions composed of hole-doped $\mathrm{La}_{2-x} \mathrm{Sr}_{x} \mathrm{CuO}_{4}$ and the parent compound $\mathrm{La}_{2} \mathrm{CuO}_{4}{ }^{7}$ They found that even isolated one-unit-cell-thick layers of $\mathrm{La}_{1.85} \mathrm{Sr}_{0.15} \mathrm{CuO}_{4}$ with the likely disorder due to doping can remain superconducting. To date, extensive theoretical studies of mechanisms for orbital selection and electron transfer in correlated oxide systems ${ }^{8-10}$ have revealed the complexity in oxide HS, where details of the interfacial electronic structure and structure distortions play decisive roles.

In this paper, we first propose a structure with a symmetrical $\mathrm{CuO}_{2}$-relative structural subunit $\left[\mathrm{SrO}-\mathrm{La}_{2} \mathrm{CuO}_{4}-\mathrm{SrO}\right]$ sandwiched between polar $\mathrm{LaAlO}_{3}$ (LAO) and nonpolar $\mathrm{SrTiO}_{3}$ (STO), which has the needed internal field for hole doping the $\mathrm{CuO}_{2}$ plane, as illustrated in the top panel of Fig. 1. This structure is studied to get some insight into the evolution from AFM state to SC state and to identify the possible role of disorder. The polarization is the ideally cleanest source with strength as high as $\sim 10^{9} \mathrm{~V} / \mathrm{m}$ and induces hole doping in the designed disorder-free HS. Here, we find that the top $\mathrm{LaO}$ plane can serve as a charge reservoir block and transfer holes to the $\mathrm{CuO}_{2}$ plane by electronic reconstruction, which occurs mainly between the La- $d_{3 z^{2}-1}$ orbital at the surface and $\mathrm{Cu}-e_{g}$ orbitals. LAO thickness and in-plane strain can be used to effectively manipulate the level of hole doping. Meantime, the HS is anticipated to have the coexistence of the AFM insulating bulk state and a surface two-dimensional electron gas (2DEG) upon judiciously tuning the LAO thickness. Remarkably, the AFM state remains in the ground state up to the $8 \%$ doping level, which reveals the possible role of chemical disorder in perturbing charge order, orbital order, and finally magnetic order in $\mathrm{La}_{2-x} \mathrm{Sr}_{x} \mathrm{CuO}_{4}$.

We carried out the numerical calculations using the Vienna ab initio Simulation Package (VASP) ${ }^{11,12}$ within the framework of the generalized gradient approximation (GGA) (PerdewBurke-Ernzerhof exchange correlation functional). ${ }^{13}$ The ionelectron interaction was modelled by the projector augmented wave (PAW) method $^{14}$ with a uniform energy cutoff of $500 \mathrm{eV}$. For the heterostructure, we used periodic boundary conditions and a vacuum layer thick enough to prohibit the out-of-plane electronic and dipole-dipole interactions between neighboring HSs. Spacing between $k$ points was $0.02 \AA^{-1}$. The geometrical structures of the HSs were optimized by employing the conjugate gradient technique, and in the final geometry, no force on the atoms exceeded $0.01 \mathrm{eV} / \AA$. In addition, we found that within the GGA the La- $5 f$ states lie only $\sim 0.5 \mathrm{eV}$ above the Fermi level, leading to a spurious mixing with the La-5d bands. Since, in practice, the La- $f$ bands lie much higher in energy ${ }^{15}$ we imposed a large $U_{f}=11 \mathrm{eV}$ and $J_{f}=0.68 \mathrm{eV}$ on the La- $f$ states. For magnetic-state calculations, we used $U_{d}=7.5 \mathrm{eV}$ and $J_{d}=0.98 \mathrm{eV}$ for $\mathrm{Cu}-d$ states, which are typical values for $\mathrm{Cu} .{ }^{16}$

We choose the lattice constant of bulk $\mathrm{SrTiO}_{3}(3.905 \AA)^{17}$ as the in-plane lattice constant $a$ of $4\left(\mathrm{SrTiO}_{3}\right) / \mathrm{La}_{2} \mathrm{Sr}_{2} \mathrm{CuO}_{6} /$ $n\left(\mathrm{LaAlO}_{3}\right)$ (hereinafter referred to as $4 \mathrm{STO} / \mathrm{LSCO} / n \mathrm{LAO}$ ) HS. Therefore, the initial Ti-O, Al-O, and $\mathrm{Cu}-\mathrm{O}$ bond lengths in the $x$ and $y$ directions become $1.952 \AA$, not far from the value in 

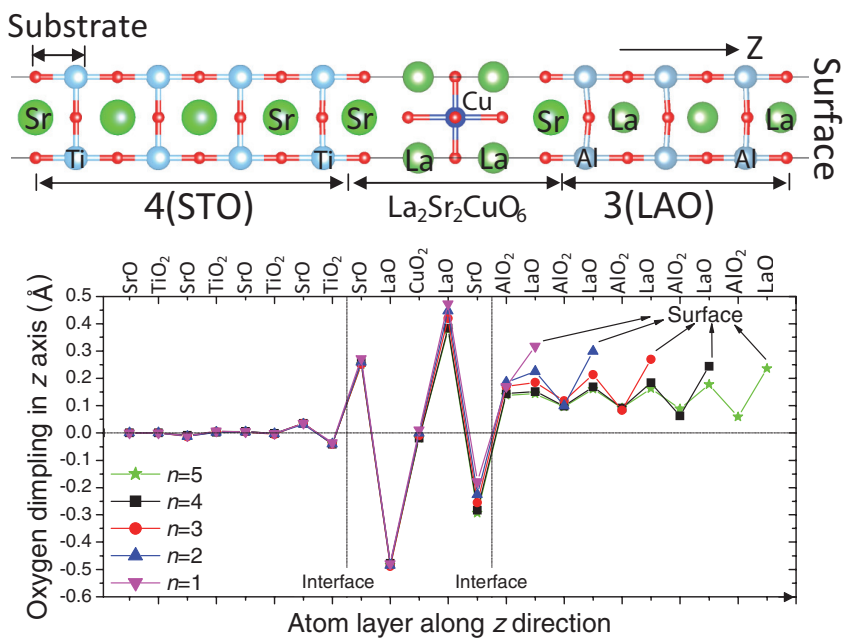

FIG. 1. (Color online) Top panel: Schematic geometrical structures of 4(STO)/ $\mathrm{La}_{2} \mathrm{Sr}_{2} \mathrm{CuO}_{6} / 3$ (LAO) HS. Bottom panel: Calculated oxygen dimpling away from cation planes for $4(\mathrm{STO}) / \mathrm{La}_{2} \mathrm{Sr}_{2} \mathrm{CuO}_{6} /$ $n$ (LAO) HSs $(1 \leqslant n \leqslant 5)$ in $z$ direction.

bulk $\mathrm{LaAlO}_{3}{ }^{17}$ or $\mathrm{La}_{2} \mathrm{CuO}_{4} \cdot{ }^{18}$ The $z$ coordinates of atoms were relaxed except for the lowest STO layer fixed for simulating substrate effects. The main effect of the relaxation, shown in the bottom panel of Fig. 1, is to make the negatively charged $\mathrm{O}$ and positively charged cations displaced relative to each other and thereby polarize the cation and anion planes. The mirror symmetry of [ $\left.\mathrm{SrO}-\mathrm{La}_{2} \mathrm{CuO}_{4}-\mathrm{SrO}\right]$ subunit retains well, and in $\mathrm{LaO}$ and $\mathrm{SrO}$ planes, oxygen atoms move symmetrically towards or against the $\mathrm{CuO}_{2}$ plane by about $0.25-0.5 \AA$, which is similar to what has been found in bulk $\mathrm{La}_{2} \mathrm{CuO}_{4}$. In the STO part, oxygen atoms in interfacial $\mathrm{SrO}$ and $\mathrm{TiO}_{2}$ planes move slightly towards interface or substrate by 0.05 $\AA$. The most important atomic polarization occurs in the LAO part, with oxygen atoms always moving towards vacuum, and the largest cation-anion polarization of $\mathrm{LaO}$ plane with $0.3 \AA$ at the surface, and the $\mathrm{AlO}_{2}$ plane with the largest polarization of $0.2 \AA$ at the interface. This polarization distortion pointing to vacuum produces a local ionic dipole moment, and together with in-plane strain it also leads to a slight decrement in the apical $\mathrm{Cu}-\mathrm{O}$ distance compared to that in the bulk $\mathrm{La}_{2} \mathrm{CuO}_{4}$.

The local ionic dipole moment arouses inevitably an internal polarized electric field in the LAO part of the HS. In order to illustrate clearly how the polar field modifies the electronic structure, we show in Fig. 2(a) the planar-averaged electrostatic potential ${ }^{19}$ and its macroscopic average (MA) along the $z$ direction perpendicular to the interface and surface of $4 \mathrm{STO} / \mathrm{LSCO} / 3 \mathrm{LAO} \mathrm{HS}$. As the figure shows, the MA potential of STO is almost flat, indicating that there is no obvious internal electric field through the STO. In the middle LSCO part, two fields with opposite polarization directions almost cancel each other out. However, in the LAO part, the MA potential is decreasing layer by layer from the interface to the surface, which reduces the valence-band edge gradually and makes the potential decrement at the LAO surface large enough to overcome the energy difference between $\mathrm{Cu}-e_{g}$ bands and La- $5 d$ bands. As a result, the conduction-band edge of the top LaO layer downshifts eventually below the Fermi level and exchanges charge with $\mathrm{Cu}-e_{g}$ bands, as seen in the

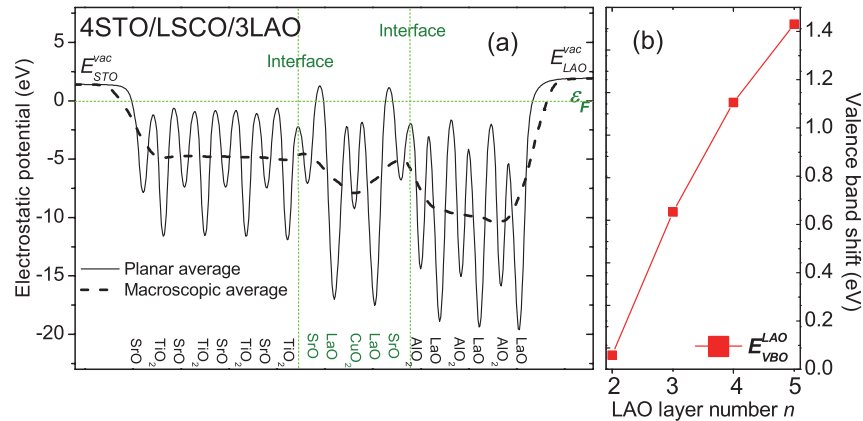

FIG. 2. (Color online) (a) Planar average and macroscopic average of electrostatic potential for metallic 4STO/LSCO/3LAO HS. Valence-band shift $E_{\mathrm{VBS}}^{\mathrm{LAO}}$ of LAO part of interface (b) for different $4 \mathrm{STO} / \mathrm{LSCO} / n \mathrm{LAO} \mathrm{HSs}$ obtained by layer-projected density of states.

layer-projected densities of states in the right panel of Fig. 3. Interestingly, for the 4STO/LSCO/2LAO HS (left panel of Fig. 3), the electron is transferred from the $\mathrm{CuO}_{2}$ plane not only to the top $\mathrm{LaO}$ layer mainly but also slightly to the interfacial STO layer, due to the smaller band gap of STO and the weak atomic polarization at the interface between STO and LSCO. Compared to the 4STO/LSCO/2LAO HS, the increased LAO thickness in the 4STO/LSCO/3LAO HS promotes the potential decrement, resulting in the enhanced internal electric field in the LAO part, so that the electron is exclusively transferred to the top LaO layer. In Fig. 3, the band edge shift layer by layer in LAO part indicates an electric field discontinuity, consistent with the accumulation of negative charge at the interface. Holes reside in the $\mathrm{CuO}_{2}$ plane inside LSCO region. The corresponding valence-band shift (VBS) of the LAO part $E_{\mathrm{VBS}}^{\mathrm{LAO}}$ [plotted in Fig. 2(b)] between valence-band edges of the interfacial and peripheral $\mathrm{LaO}$ planes, increases with increasing thickness of the LAO part. Based on the
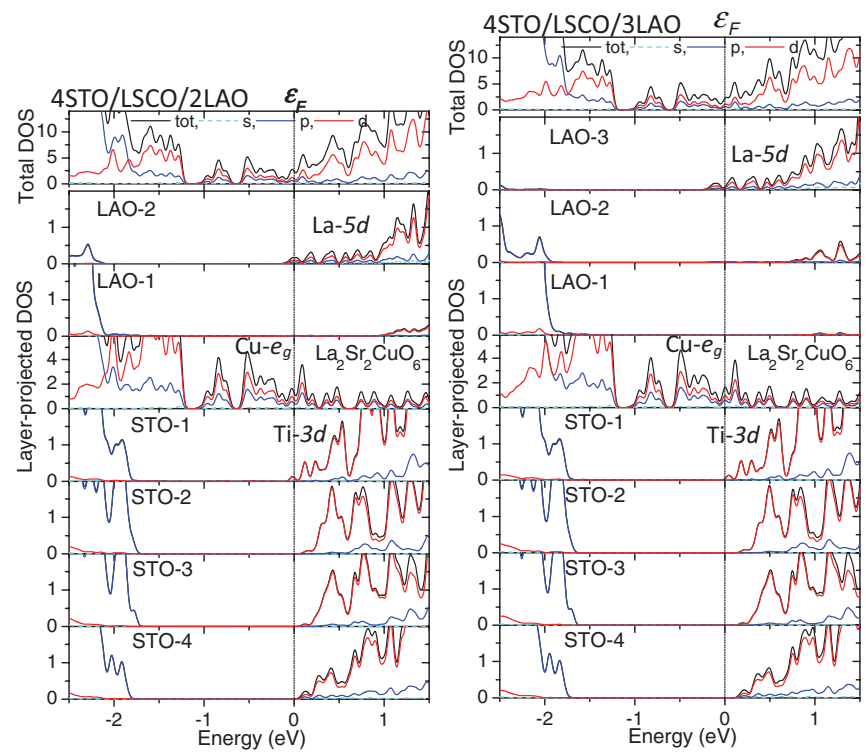

FIG. 3. (Color online) Layer-projected density of states of 4STO/LSCO/2LAO (left panel) and 4STO/LSCO/3LAO (right panel) HSs from GGA calculations. 


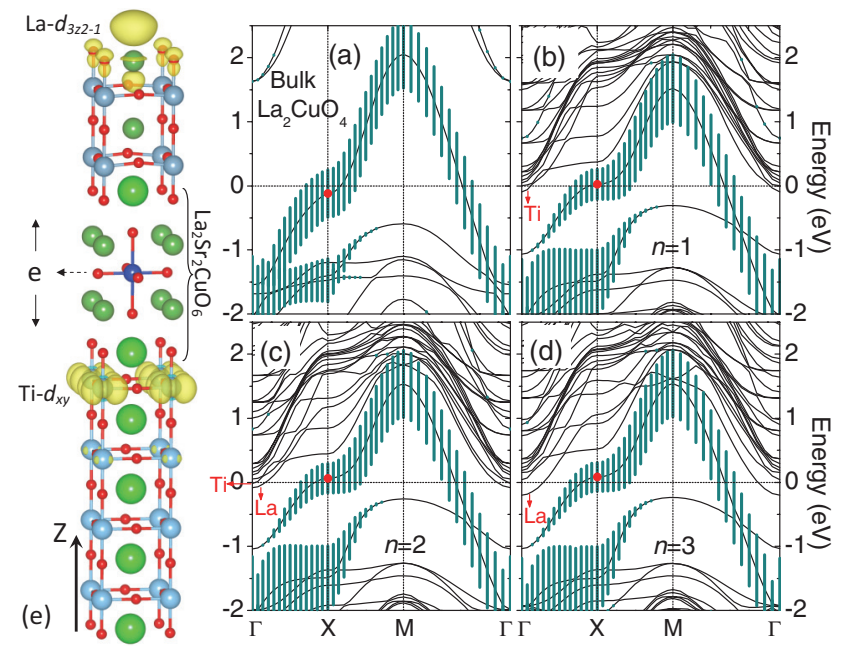

FIG. 4. (Color online) GGA band structures of (a) bulk $\mathrm{La}_{2} \mathrm{CuO}_{4}$, (b) $4 \mathrm{STO} / \mathrm{LSCO} / 1 \mathrm{LAO}$, (c) $4 \mathrm{STO} / \mathrm{LSCO} / 2 \mathrm{LAO}$, and (d) $4 \mathrm{STO} / \mathrm{LSCO} / 3 \mathrm{LAO} \mathrm{HSs}$. The Fermi level $\varepsilon_{F}$ is set at zero. (e) The $0.04 \AA^{-3}$ partial charge density isosurfaces for the two partially occupied conduction bands (Ti-3d and La- $5 d$ ) at the $\Gamma$ point for $4 \mathrm{STO} / \mathrm{LSCO} / 2 \mathrm{LAO} \mathrm{HS}$.

$E_{\mathrm{VBS}}^{\mathrm{LAO}}$ values of $4 \mathrm{STO} / \mathrm{LSCO} / 4 \mathrm{LAO}$ and $4 \mathrm{STO} / \mathrm{LSCO} / 5 \mathrm{LAO}$ HSs, we estimate the internal electric field to be $85.8 \mathrm{mV} / \AA$, consistent with the experimentally observed $80.1 \mathrm{mV} / \AA$ for the LAO/STO HS. ${ }^{20}$

An evolution of surface states (La- $d$ ) and/or interface states (Ti- $d)$ can be clearly observed in electronic structures of HSs. Figures 4(b)-4(d) show GGA energy bands of $4 \mathrm{STO} / \mathrm{LSCO} / 1 \mathrm{LAO}, 4 \mathrm{STO} / \mathrm{LSCO} / 2 \mathrm{LAO}$, and 4STO/LSCO/3LAO HSs in a $4.5 \mathrm{eV}$ region around the Fermi level $\varepsilon_{F} \equiv 0$ and along the symmetry lines $\Gamma-\mathrm{X}-\mathrm{M}-\Gamma=(0,0,0)-\left(\frac{\pi}{a}, 0,0\right)-\left(\frac{\pi}{a}, \frac{\pi}{a}, 0\right)-(0,0,0)$. The energy bands of bulk $\mathrm{La}_{2} \mathrm{CuO}_{4}$ is also plotted in Fig. 4(a) for comparison. For three HSs, electronic properties around $\varepsilon_{F}$ are still mainly controlled by the $\mathrm{Cu}-e_{g}$ bands, which are above the filled $\mathrm{O}-2 p$ and $\mathrm{Cu}-t_{2 g}$ bands and below the slightly filled Ti-3 $d$ and/or La-5 $d$ bands. Here, due to the deformed geometrical structure and the quantum confinement from the finite-size effect, the high degeneracy of the original bulk Ti-3d and La-5d bands is lift, and band splitting occurs in the HS. We plot the $\mathrm{Cu}-d_{x^{2}-y^{2}}$ fatband around $\varepsilon_{F}$ to disclose its orbital contribution. Compared to energy bands of bulk $\mathrm{La}_{2} \mathrm{CuO}_{4}$, the $\mathrm{Cu}-e_{g}$ band width becomes smaller due to in-plane strain. Both $\mathrm{Cu}-e_{g}$ bands exhibit an obvious band upshift, resulting in a significant polarization-induced hole doping. The representative character is that the upper $\mathrm{Cu}-e_{g}$ band along $\Gamma \mathrm{X}$ now becomes partially occupied [see the relative location of the red point around $\varepsilon_{F}$ in Figs. 4(a)-4(d)]. It is worth noting that there exists a weak competition between Ti- $3 d$ and $\mathrm{La}-5 d$ to catch electrons from $\mathrm{Cu}-e_{g}$. We find that there is only $\mathrm{Ti}-3 d(\mathrm{La}-5 d)$ band transferring charge with the $\mathrm{Cu}-e_{g}$ band in 4STO/LSCO/1LAO (4STO/LSCO/3LAO) HS. However, in the $4 \mathrm{STO} / \mathrm{LSCO} / 2 \mathrm{LAO}$ case, both kinds of bands touch the Fermi level at the $\Gamma$ point. Therefore, the changing of LAO thickness from 1 to 3 enhances internal polarization in favor of single directional charge transfer between $\mathrm{Cu}-e_{g}$ and La-5d.
In order to investigate the microscopic orbital physics of polarization-induced hole doping, we plot the typical partial charge density of the two partially occupied conduction bands (Ti-3 $d$ and La-5d) of the 4 STO/LSCO/2LAO HS at the $\Gamma$ point in Fig. 4(e). Obviously, hole doping originates from charge transfer between $\mathrm{Cu}-e_{g}$ orbitals and La- $d_{3 z^{2}-1}\left(\mathrm{Ti}-d_{x y}\right)$ at the surface (interface), coinciding with the trend of electronic structures in Figs. 3 and 4(c). Here, La- $d_{3 z^{2}-1}$ has considerable hybridization with the in-plane oxygen atoms' $p_{z}$ orbitals due to the strong covalent effect. Structural analysis of the peripheral LAO indicates that the coordination sphere around the La ion is eight coordinated with four oxygen atoms coming from the $\mathrm{LaO}$ plane, and four other oxygen atoms coming from the adjacent $\mathrm{AlO}_{2}$ plane. The geometry around the La cation in the top $\mathrm{LaO}$ plane is best described as square-antiprismatic, in which La- $d_{3 z^{2}-1}$ becomes nondegenerate and is the lowest conduction band at the $\Gamma$ point in Figs. 4(c) and 4(d). At the interface, the Ti- $d_{x y}$ bands are lower than the Ti- $d_{x z}, d_{y z}$ bands due to the larger in-plane hopping integrals. Interestingly, the occupied La- $d_{3 z^{2}-1}\left(\mathrm{Ti}-d_{x y}\right)$ state at the $\Gamma$ point is mainly located around the surface (interface) and far away from the $\mathrm{CuO}_{2}$ plane, as indicated in Fig. 4(e) and from analysis of the wave function characteristics, which favors the realization of possible $\mathrm{SC}$ in the $\mathrm{CuO}_{2}$ plane. To quantify the hole doping level, we calculate the amount of hole in the $\mathrm{Cu}-e_{g}$ orbitals for $4 \mathrm{STO} / \mathrm{LSCO} / n \mathrm{LAO}$ with $n$ varying from 1 to 5 . As we can see in Fig. 5(a), hole doping rises from $2.8 \%$ to $6.6 \%$ steadily with increasing LAO thickness. Accordingly, the energy value of the partially occupied upper $\mathrm{Cu}-e_{g}$ band at the $\mathrm{X}$ point upshifts gradually in Fig. 5(b), which is consistent with the foregoing understanding of electronic structures plotted in Figs. 3 and 4(a)-4(d).

We further investigate the effect of in-plane strain on the doping level because electronic properties exhibit remarkable coupling with the lattice degree of freedom in perovskite materials. Similar calculations are made for $4 \mathrm{STO} / \mathrm{LSCO} / 4 \mathrm{LAO}$ HS with two different in-plane lattice constants of 4.005 and $3.79 \AA$. We find that tensile strain can effectively increase the

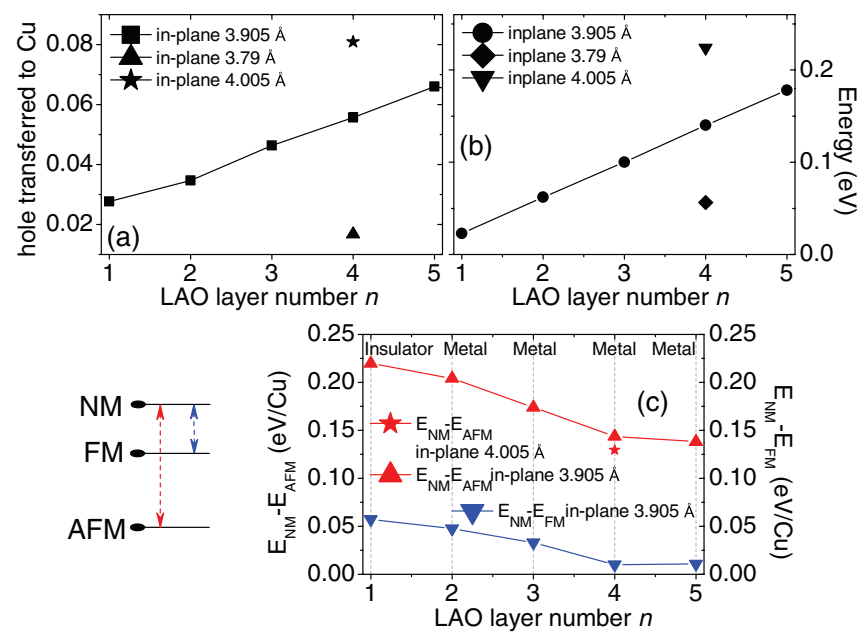

FIG. 5. (Color online) (a) The amount of hole in $\mathrm{Cu}-e_{g}$ orbitals, (b) energy of the partially occupied upper $\mathrm{Cu}-e_{g}$ band at the $\mathrm{X}$ point, and (c) energy differences $E_{\mathrm{NM}}-E_{\mathrm{AFM}}$ and $E_{\mathrm{NM}}-E_{\mathrm{FM}}$ as a function of LAO layer number $n$ in HS. 


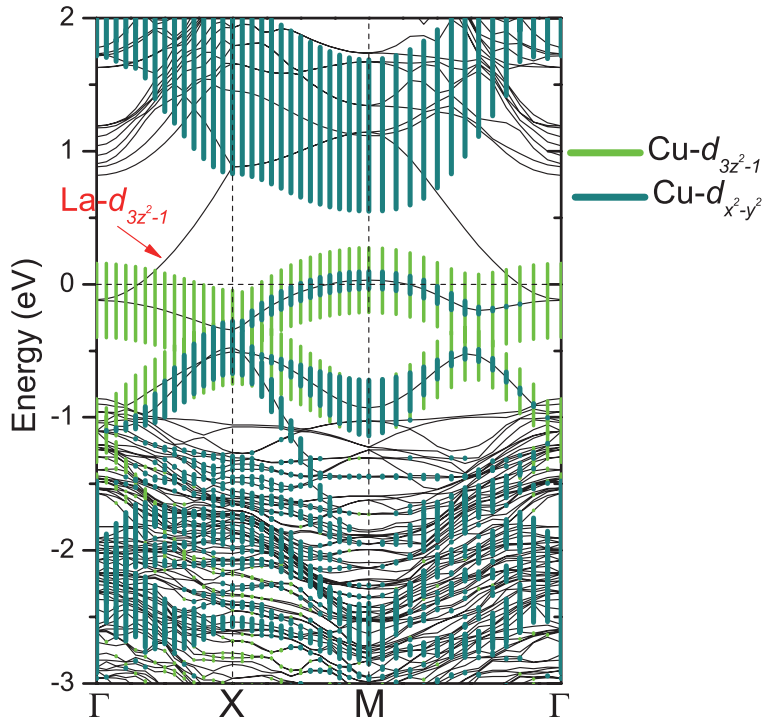

FIG. 6. (Color online) $\mathrm{GGA}+U_{d} \mathrm{AFM}$ band structure of 4STO/LSCO/3LAO HS. The Fermi level $\varepsilon_{F}$ is set at zero.

doping level to $8 \%$, but compressive strain decreases it to $1.7 \%$ [see Fig. 5(a)]. The energy value of the partially occupied upper $\mathrm{Cu}-e_{g}$ band at the $\mathrm{X}$ point also has a corresponding upshift or downshift in Fig. 5(b). Thus, the tuning of the in-plane lattice constant can serve as an effective way to manipulate the polarization-induced hole doping level.

Next, we need to know the stability of magnetic states in this hole-doped HS without chemically introduced disorder. We calculated the energy difference in terms of $E_{\mathrm{NM}}-E_{\mathrm{AFM}}$ and $E_{\mathrm{NM}}-E_{\mathrm{FM}}$ under different doping levels [see Fig. 5(c)] by introducing the Coulomb correlation $U_{d} .{ }^{21}$ The $E_{\mathrm{NM}}-E_{\mathrm{AFM}}$ decreases gradually from $0.22 \mathrm{eV} / \mathrm{Cu}$ at $n=1$ to $0.14 \mathrm{eV} / \mathrm{Cu}$ at $n=5$, which is smaller than the $0.21 \mathrm{eV} / \mathrm{Cu}$ in bulk $\mathrm{La}_{2} \mathrm{CuO}_{4}$. Meantime, the $E_{\mathrm{NM}}-E_{\mathrm{FM}}$ varies from $0.06 \mathrm{eV} / \mathrm{Cu}$ at $n=1$ to $0.01 \mathrm{eV} / \mathrm{Cu}$ at $n=5$, which is also smaller than the $0.03 \mathrm{eV} / \mathrm{Cu}$ in bulk $\mathrm{La}_{2} \mathrm{CuO}_{4}$. Both $E_{\mathrm{NM}}-E_{\mathrm{AFM}}$ and $E_{\mathrm{NM}}-E_{\mathrm{FM}}$ vary much more weakly upon increasing LAO thickness above four. Even for $\sim 8 \%$ doping at $n=4$ under tensile strain $(4.005 \AA), E_{\mathrm{NM}}-E_{\mathrm{AFM}}$ only slightly changes to $0.13 \mathrm{eV} / \mathrm{Cu}$. In addition, strong electronic correlation leads to an insulator-metal transition occurring at the critical thickness $n=2$ due to the charge transfer revealed in the GGA bands. Taking 4STO/LSCO/3LAO HS as an example, the AFM band structure with $\mathrm{Cu}-e_{g}$ orbital characteristics in Fig. 6 shows clearly that both $e_{g}$ orbitals are involved in the charge transfer process. Different from the situation in bulk hole-doped $\mathrm{La}_{2} \mathrm{CuO}_{4}$ with introduced disorder, disorder is absent in heterostructures and therefore is the reason for the unexpected stable AFM state. Based on the above discussions, we conclude that disorder itself is an important factor for the realization of SC through perturbing the charge order, orbital order, and antiferromagnetic long range order. Further research in this direction is needed to gain better understanding of the delicate evolution among intriguing quantum states.

In addition, consistent with the GGA result shown in Fig. 4(d), only one La- $d_{3 z^{2}-1}$ band emerges in the AFM band gap of the $\mathrm{CuO}_{2}$ plane and crosses the Fermi level (Fig. 6). This indicates that a strongly localized 2DEG forms at the $\mathrm{LaO}$ surface [see Fig. 4(e)], which is promising for the development of novel applications in nanoscale oxide electronics. Motivated by real experiments for STO/LAO/STO HSs, ${ }^{22}$ in which an additional STO capping layer is found to prevent atomic reconstruction at the LAO surface and trigger the electronic reconstruction at a significantly lower LAO film thickness than for the uncapped systems. Hence, we here investigate the effect of the STO capping layer on the charge transfer in our $4 \mathrm{STO} / \mathrm{LSCO} / 5 \mathrm{LAO} \mathrm{HS}$. Our simulations reveal dramatic modification of charge transfer. Instead of La- $d_{3 z^{2}-1}$, Ti- $t_{2 g}$ orbitals of the capping layer exchange holes with electrons of $\mathrm{Cu}-e_{g}$ orbitals not only in SrO-terminated 4STO/LSCO/5LAO/1STO HSs but also in $\mathrm{TiO}_{2}$-terminated 4STO/LSCO/5LAO/1.5STO HSs. The hole concentration in the $\mathrm{CuO}_{2}$ plane remains almost the same after adding the capping layer.

In conclusion, our analysis indicates that a polarizationinduced hole doping of the $\mathrm{CuO}_{2}$ plane and an unexpected coexistence of an antiferromagnetic insulating bulk state and a surface 2DEG are present in the proposed disorder-free heterostructure with a single copper-oxygen plane. The polarized potential of the LAO part makes La- $d_{3 z^{2}-1}$ bands at the surface overlap with $\mathrm{Cu}-e_{g}$ bands, which is the origin of an controllable hole doping and metallic surface state. The hole concentration can be rationally manipulated by tuning the $\mathrm{LaAlO}_{3}$ thickness and in-plane strain. STO-capped STO/LSCO/LAO has the similar charge transport mechanism, instead of $\mathrm{La}-d_{3 z^{2}-1}$, Ti- $t_{2 g}$ orbitals of capping layer exchange holes with electrons of $\mathrm{Cu}-e_{g}$ orbitals. The remarkable stable AFM state up to $8 \%$ doping sheds a light on the possible important effect of chemical disorder on AFM instability and SC formation in bulk hole-doped $\mathrm{La}_{2} \mathrm{CuO}_{4}$.

We are grateful for the interesting discussions with T. Maurice Rice, Ole Krogh Andersen, Fuchun Zhang, Haiqing Lin, Hans Hilgenkamp, Jaichan Lee, Adrian Gozar, and Daoxin Yao. This work was supported in part by the A*STAR SERC Grant No. 1121202012.

\footnotetext{
*hbsu@ntu.edu.sg

${ }^{1}$ J. G. Bednorz and K. A. Müller, Z. Phys. B: Condens. Matter 64, 189 (1986).

${ }^{2}$ P. W. Andersen, Science 235, 1196 (1987).

${ }^{3}$ K. H. Höglund, A. W. Sandvik, and S. Sachdev, Phys. Rev. Lett. 98, 087203 (2007); K. H. Höglund and A. W. Sandvik,
}

Phys. Rev. B 70, 024406 (2004); Dao-Xin Yao, J. Gustafsson, E. W. Carlson, and A. W. Sandvik, ibid. 82, 172409 (2010).

${ }^{4}$ A. Ohtomo and H. Y. Hwang, Nature (London) 427, 423 (2004);

S. Thiel, G. Hammerl, A. Schmehl, C. W. Schneider, and

J. Mannhart, Science 313, 1942 (2006); N. Reyren, S. Thiel,

A. D. Caviglia, L. F. Kourkoutis, G. Hammerl, C. Richter, 
C. W. Schneider, T. Kopp, A.-S. Rüetschi, D. Jaccard, M. Gabay, D. A. Muller, J.-M. Triscone, and J. Mannhart, ibid. 317, 1196 (2007); M. Huijben, A. Brinkman, G. Koster, G. Rijnders, H. Hilgenkamp, and D. H. A. Blank, Adv. Mater. 21, 1665 (2009).

${ }^{5}$ J. Chakhalian, J. W. Preeland, G. Srajer, J. Strempfer, G. Khaliullin, J. C. Cezar, T. Charlton, R. Dalgliesh, C. Bernhard, G. Cristiani, H.-U. Habermeier, and B. Keimer, Nat. Phys. 2, 244 (2006); J. Chakhalian, J. W. Preeland, H.-U. Habermeier, G. Cristiani, G. Khaliullin, M. van Veenendaal, and B. Keimer, Science 318, 1114 (2007).

${ }^{6}$ H. W. Jang, D. A. Felker, C. W. Bark, Y. Wang, M. K. Niranjan, C. T. Nelson, Y. Zhang, D. Su, C. M. Folkman, S. H. Baek, S. Lee, K. Janicka, Y. Zhu, X. Q. Pan, D. D. Fong, E. Y. Tsymbal, M. S. Rzchowski, and C. B. Eom, Science 331, 886 (2011).

${ }^{7}$ I. Bozovic, G. Logvenov, M. A. J. Verhoeven, P. Caputo, E. Goldobin, and T. H. Geballe, Nature (London) 422, 873 (2003); G. Logvenov, A. Gozar, and I. Bozovic, Science 326, 699 (2009); A. T. Bollinger, G. Dubuis, J. Yoon, D. Pavuna, J. Misewich, and I. Bozovic, Nature (London) 472, 458 (2011).

${ }^{8}$ V. I. Anisimov, I. A. Nekrasov, D. E. Kondakov, T. M. Rice, and M. Sigrist, Eur. Phys. J. B 25, 191 (2002); A. Koga, N. Kawakami, T. M. Rice, and M. Sigrist, Phys. Rev. Lett. 92, 216402 (2004); R. Arita and K. Held, Phys. Rev. B 72, 201102(R) (2005); A. I. Poteryaev, J. M. Tomczak, S. Biermann, A. Georges, A. I. Lichtenstein, A. N. Rubtsov, Tanusri Saha-Dasgupta, and O. K. Andersen, ibid. 76, 085127 (2007).

${ }^{9}$ P. Hansmann, Xiaoping Yang, A. Toschi, G. Khaliullin, O. K. Andersen, and K. Held, Phys. Rev. Lett. 103, 016401 (2009); P. Hansmann, A. Toschi, Xiaoping Yang, O. K. Andersen, and K. Held, Phys. Rev. B 82, 235123 (2010).
${ }^{10}$ Xiaoping Yang and Haibin Su, ACS Appl. Mater. Interfaces 3, 3819 (2011).

${ }^{11}$ G. Kresse and J. Hafner, Phys. Rev. B 47, 558 (1993); 49, 14251 (1994).

${ }^{12}$ G. Kresse and J. Furthmüller, Phys. Rev. B 54, 11169 (1996); Comput. Mater. Sci. 6, 15 (1996).

${ }^{13}$ J. P. Perdew, K. Burke, and M. Ernzerhof, Phys. Rev. Lett. 77, 3865 (1996).

${ }^{14}$ G. Kresse and D. Joubert, Phys. Rev. B 59, 1758 (1999); P. E. Blöchl, ibid. 50, 17953 (1994).

${ }^{15}$ M. T. Czyzyk and G. A. Sawatzky, Phys. Rev. B 49, 14211 (1994).

${ }^{16}$ V. I. Anisimov, J. Zaanen, and O. K. Andersen, Phys. Rev. B 44, 943 (1991).

${ }^{17}$ J. Zhu, L. Zheng, Y. Zhang, X. H. Wei, W. B. Luo, and Y. R. Li, Mater. Chem. Phys. 100, 451 (2006).

${ }^{18}$ J. D. Jorgensen, H.-B. Schüttler, D. G. Hinks, D. W. Capone II, K. Zhang, M. B. Brodsky, and D. J. Scalapino, Phys. Rev. Lett. 58, 1024 (1987).

${ }^{19}$ A. Baldereschi, S. Baroni, and R. Resta, Phys. Rev. Lett. 61, 734 (1988).

${ }^{20}$ G. Singh-Bhalla, C. Bell, J. Ravichandran, W. Siemons, Y. Hikita, S. Salahuddin, A. F. Hebard, H. Y. Hwang, and R. Ramesh, Nat. Phys. 7, 80 (2011).

${ }^{21}$ Coulomb correction effect on active La- $d$ or Ti- $d$ states is found to be very weak due to their low carrier density (far away from half filling), therefore no significant change occurs in the obtained electronic structures.

${ }^{22}$ R. Pentcheva, M. Huijben, K. Otte, W. E. Pickett, J. E. Kleibeuker, J. Huijben, H. Boschker, D. Kockmann, W. Siemons, G. Koster, H. J. W. Zandvliet, G. Rijnders, D. H. A. Blank, H. Hilgenkamp, and A. Brinkman, Phys. Rev. Lett. 104, 166804 (2010). 